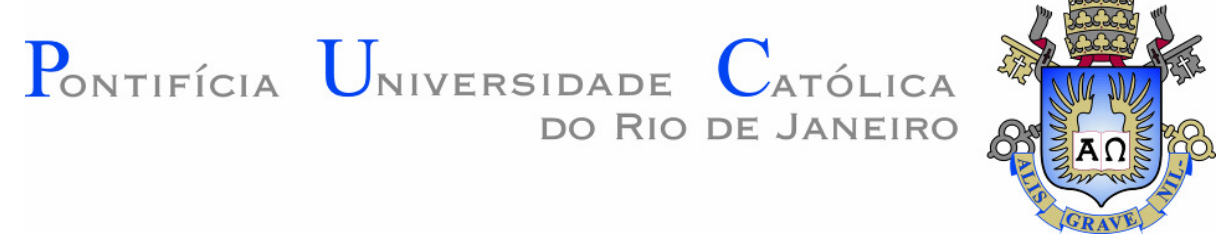

Giancarlo Luis Gómez Gonzáles

\title{
Aplicação da Técnica SIFT para Determinação de Campos de Deformações de Materiais usando Visão Computacional
}

Dissertação de Mestrado

Dissertação apresentada como requisito parcial para obtenção do título de Mestre pelo Programa de PósGraduação em Engenharia Mecânica da PUC-Rio.

Orientador: Prof. Marco Antonio Meggiolaro

Rio de Janeiro setembro de 2010 


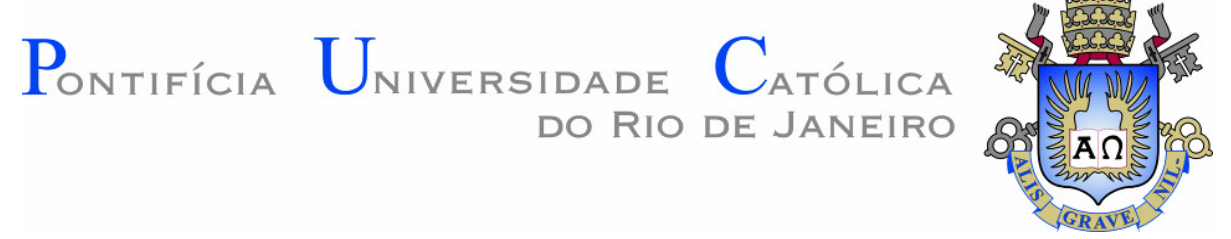

Giancarlo Luis Gómez Gonzáles

\title{
Aplicação da Técnica SIFT para Determinação de Campos de Deformações de Materiais usando Visão Computacional
}

\begin{abstract}
Dissertação apresentada como requisito parcial para obtenção do título de Mestre pelo Programa de PósGraduação em Engenharia Mecânica da PUC-Rio. Aprovada pela Comissão Examinadora abaixo assinada.
\end{abstract}

Prof. Marco Antonio Meggiolaro

Orientador

Departamento Engenharia Mecânica PUC-Rio

Prof. Jaime Tupiassú Pinho de Castro

Departamento Engenharia Mecânica PUC-Rio

Prof. Raul Queiroz Feitosa

Departamento de Engenharia Elétrica PUC-Rio

Prof. José Eugenio Leal

Coordenador Setorial do Centro Técnico Científico - PUC-Rio 
Todos os direitos reservados. É proibida a reprodução total ou parcial do trabalho sem autorização da universidade, do autor e do orientador.

\section{Giancarlo Luis Gómez Gonzáles}

Graduado em Engenharia Eletrônica pela Universidad Nacional de San Agustín, Arequipa - Perú, em 2006.

Ficha Catalográfica

\section{Gómez Gonzáles, Giancarlo Luis}

Aplicação da técnica SIFT para determinação de campos de deformações de materiais usando visão computacional / Giancarlo Luis Gómez Gonzáles ; orientador: Marco Antonio Meggiolaro. -2010.

109 f. : il. (color.); $30 \mathrm{~cm}$

Dissertação (Mestrado)-Pontifícia Universidade Católica do Rio de Janeiro, Rio de Janeiro, Departamento de Engenharia Mecânica, 2010.

Inclui bibliografia.

1. Engenharia mecânica - Teses. 2. SIFT. 3. Campos de deformações. 4. Visão computacional. 5. Problemas inversos. I. Meggiolaro, Marco Antonio. II. Pontifícia Universidade Católica do Rio de Janeiro. Departamento de Engenharia Mecânica. III. Título.

CDD: 621 
Com muito carinho e afeto dedico este meu trabalho à minha família, meu irmão Jonathan, minha irmã Yelka e a aos meus pais, Luis e Mariela, pela fé, confiança e amor. 


\section{Agradecimentos}

Muitos me ajudaram na realização deste trabalho, direta ou indiretamente, estando de alguma forma presente nestes dois anos que se passaram. Aos que se fizeram notáveis, seguem meus agradecimentos.

A Deus, que nunca abandona.

Ao meu orientador Professor Marco Antonio Meggiolaro, pelos diversos ensinamentos e ajuda no processo de elaboração do presente trabalho, sem o que não teria sido possível concluir esta dissertação.

Aos professores do curso, em especial aos Professores Jaime Tupiassú, Raúl Feitosa, Marley Velasco pelos ensinamentos e ajuda quando deles precisei.

À Pontifícia Universidade Católica de Rio de Janeiro e ao Curso de PósGraduação em Engenharia Mecânica, pelo ambiente acadêmico-científico favorável ao desempenho deste trabalho.

À Coordenação de Aperfeiçoamento de Pessoal de Nível Superior - CAPES pelo fomento financeiro no Brasil.

Aos amigos e colegas do curso pelo conhecimento dividido, companheirismo, momentos de stress e diversão.

Aos amigos de sempre Alessandro e Gustavo, obrigado pela incondicional amizade.

À minha amiga Paula pelas acertadas aulas de português e pelo desprendido suporte e amizade.

E finalmente, á pessoa mais especial, aquela que é a direção e principal motor da minha vida, Luciana. 


\section{Resumo}

Gómez Gonzáles, Giancarlo Luis; Meggiolaro, Marco Antonio. Aplicação da Técnica SIFT para Determinação de Campos de Deformações de Materiais usando Visão Computacional. PUC-Rio, 2010. 109p. Dissertação de Mestrado - Departamento de Engenharia Mecânica, Pontifícia Universidade Católica do Rio de Janeiro.

Esta dissertação apresenta uma metodologia para medição visual de campos de deformações (2D) em materiais, por meio da aplicação da técnica SIFT (Scale Invariant Feature Transform). As imagens analisadas são capturadas por uma câmera digital em estágios diferentes durante o processo de deformação de um material quando este é submetido a esforços mecânicos. SIFT é uma das técnicas modernas de visão computacional e um eficiente filtro para extração e descrição de pontos de características relevantes em imagens. Estes pontos de interesse são largamente invariantes a transformações em escala, iluminação e rotação. A metodologia é baseada no cálculo do gradiente de funções que representam o correspondente campo de deformações em um material durante um ensaio mecânico sob diferentes condições de contorno. As funções são calibradas com a aplicação da análise inversa sobre o conjunto de pontos homólogos de duas imagens extraídos pelo algoritmo SIFT. A formulação da solução ao problema inverso combina os dados experimentais fornecidos pelo SIFT e o método linear de mínimos quadrados para estimação dos parâmetros de deformação. Os modelos propostos para diferentes corpos de prova são avaliados experimentalmente com a ajuda de extensômetros para medição direta das deformações. O campo de deformações identificado pelo sistema de visão computacional é comparado com os valores obtidos pelos extensômetros e por simulações feitas no programa de Elementos Finitos ANSYS. Os resultados obtidos mostram que o campo de deformações pode ser medido utilizando a técnica SIFT, gerando uma nova ferramenta visual de medição para ensaios mecânicos que não se baseia nas técnicas tradicionais de correlação de imagens.

\section{Palavras-chave}

SIFT; campos de deformações; visão computacional; processamento de imagens; problemas inversos. 


\section{Abstract}

Gómez Gonzáles, Giancarlo Luis; Meggiolaro, Marco Antonio. Application of the SIFT Technique to Determinate Strain Fields of Materials using Computer Vision. PUC-Rio, 2010. 109p. M.Sc. Dissertation Departamento de Engenharia Mecânica, Pontifícia Universidade Católica do Rio de Janeiro.

This thesis presents a methodology for measurement of strain fields in materials by applying the SIFT technique (Scale Invariant Feature Transform). The images analyzed are captured by a digital camera at different stages during the deformation process of a material when it is subjected to mechanical stress. SIFT is one of the modern computer vision techniques and an efficient filter for extraction and description of relevant feature points in images. These interest points are largely invariant to changes in scale, illumination and rotation. The methodology is based on the calculation of the gradient of the functions that represents the corresponding strain field in the material during a mechanical test under different boundary conditions. The functions are calibrated with the application of inverse analysis on the set of homologous points of two images extracted by the SIFT algorithm. The formulation of the solution to the inverse problem combines the experimental data processed by the SIFT and linear least squares method for the estimation of strain parameters. The proposed models for different specimens are evaluated experimentally with strain gauges for direct measurement of the deformations. The strain field identified by the computer vision system is compared with values obtained by strain gauges and simulations with the ANSYS finite element program. The proposed models for different types of measurements are experimentally evaluated with strain gages, including the estimation of mechanical properties. The results show that the strain field can be measured using the SIFT technique, developing a new visual tool for measurement of mechanical tests that are not based on traditional techniques of image correlation.

\section{Keywords}

SIFT; strain field; computer vision; image processing; inverse problems. 


\section{Sumário}

1 INTRODUÇÃO 16

1.1. Motivação 19

1.2. Metodologia 20

1.3. Objetivos 23

1.3.1. Objetivo Geral 23

1.3.2. Objetivos Específicos 23

1.4. Organização da Dissertação 24

2 CONCEITOS TEÓRICOS FUNDAMENTAIS 25

2.1. Princípios da Teoria da Elasticidade 25

2.1.1. Estado de Tensão 25

2.1.2. Estado de Deslocamento e Deformação 27

2.1.3. Componentes de Deformação em Coordenadas Cartesianas 28

2.1.4. Componentes de Deformação em Coordenadas Polares 31

2.2. Concentração de Tensões 32

2.2.1. Distribuição de Tensões da Placa infinita com Furo Circular 33

2.3. Solução de Mínimos Quadrados de Sistemas Lineares 35

2.3.1. Interpretação Geométrica 36

2.3.2. Cálculo da Solução por Mínimos Quadrados 36 
3.1. Técnicas Interferométricas 38

3.1.1. Interferometria Digital de Speckle (ESPI) 38

3.1.2. Fontes de Erro na Medição com Interferometria 40

3.2. Técnicas de Correlação Digital 40

3.2.1. Correlação Digital de Imagens 40

3.2.2. Correlação Digital de Speckles Interferométricos 43

3.2.3. Problemas na Correlação Digital 44

3.3. Visão Computacional para Análise de Deformações 45

4 SIFT (Scale Invariant Feature Transform) 48

4.1. Etapas do Algoritmo SIFT 48

4.1.1. Detecção de Extremos 48

4.1.2. Localização Precisa de Pontos Chaves 51

4.1.3. Atribuição da Orientação dos Descritores 54

4.1.4. Construção do Descritor Local 56

4.2. Matching: Encontrando os Pontos em Comum 59

5 METODOLOGIA 61

5.1. Preparação do Corpo de Prova 62

5.2. Aquisição das Imagens 65

5.3. Pós-Processamento das Imagens 66

5.4. Determinação do Campo de Deformações 68

5.4.1. Estimativa da Função Deslocamento 68 
5.4.2. Determinação dos Parâmetros de Deformação 70

5.4.3. Solução Aproximada por Mínimos Quadrados 71

5.4.4. Cálculo da Solução de Mínimos Quadrados 73

5.5. Campo de Deformações em uma Placa com Furo Circular 73

5.5.1. Identificação dos Parâmetros de Deformação 73

5.5.2. Deslocamentos na Placa com Furo 75

5.5.3. Solução Aproximada por Mínimos Quadrados 77

6 SIMULAÇÕES

6.1. Campo de Deformações Uniforme 81

6.2. Campo de Deformações Variável 82

6.3. Campo de Deformações de uma Placa com Furo Central 84

7 RESULTADOS EXPERIMENTAIS 90

7.1. Determinação do Módulo de Elasticidade 90

$\begin{array}{ll}\text { 7.1.1. Resultados } & 91\end{array}$

7.2. Determinação do Coeficiente de Poisson 95

$\begin{array}{ll}\text { 7.2.1. Resultados } & 97\end{array}$

$\begin{array}{ll}\text { 7.3. Considerações Gerais } & 100\end{array}$

8 CONCLUSÕES E TRABALHOS FUTUROS 102

9 REFERENCIAS BIBLIOGRÁFICAS 104 


\section{Lista de figuras}

Figura 2.1 Elemento cúbico sujeito a tensões nas faces (Timoshenko \& Goodier, 1970).

Figura 2.2 Estrutura original e deformada (Fonte: Laier e Barreiro, 1983).

Figura 2.3 Elemento infinitesimal de um corpo elástico (Timoshenko \& Goodier, 1970).

Figura 2.4 Deformações no plano xy (Timoshenko \& Goodier, 1970).

Figura 2.5 Deslocamentos em coordenadas polares $x y$ (Timoshenko \& Goodier, 1970).

Figura 2.6 Geometria de uma placa infinita com um furo circular cilíndrico submetido a uma tensão remotamente uniforme.

Figura 2.7 Distribuição de $\sigma_{r}(r, \pi / 2)$ e de $\sigma_{\theta}(r, \pi / 2)$ (Castro \& Meggiolaro, 2009).

Figura 2.8 Interpretação geométrica da solução por mínimos quadrados (Anton \& Rorres, 2001).

Figura 3.1 Padrão de granulado característico (esquerda), deformado (centro) e franjas de correlações resultantes da superposição dos granulados característicos e deformado (Pires et al., 2006).

Figura 3.2 Típica montagem de um sistema de interferometria.

Figura 3.3 Montagem experimental para DIC (Lecompte, 2006).

Figura 3.4 Diagrama esquemático do funcionamento do algoritmo de Correlação Digital de Imagens.

Figura 3.5 Montagem experimental para DSCM (Guo et al., 2008). 
Figura 4.1 Exemplos de aplicação dos filtros. Imagem de padrão de granulados (esquerda), filtro Gaussiano (centro), e resultado da Diferença de Gaussianas (direita).

Figura 4.2 Representação do procedimento de obtenção das Diferenças de Gaussianas DoG para diversas oitavas de uma imagem. (Lowe 2004).

Figura 4.3 Detecção de extremos no espaço-escala (Lowe, 2004).

Figura 4.4 Pontos-chaves localizados em duas imagens, antes e após uma deformação trativa na direção vertical

Figura 4.5 Histograma de orientações de um ponto-chave.

Figura 4.6 Atribuição de orientação e magnitude a cada ponto-chave.

Figura 4.7 Mapa de gradientes para $n=2$ regiões e $k=4$ pixels. (Lowe, 2004).

Figura 4.8 Construção do descritor para um ponto-chave de $2 \times 2$ com 48 elementos (Lowe, 2004).

Figura 4.9 Processo de correspondência entre duas imagens através da técnica SIFT.

Figura 5.1 Programa desenvolvido na plataforma Matlab®.

Figura 5.2 Superfície arranhada (esquerda) e pontos-chave / gradientes localizados pelo SIFT (direita).

Figura 5.3 Superfície preta pulverizada com tinta branca (esquerda) e pontos-chaves / gradientes localizados pelo SIFT (direita).

Figura 5.4 Montagem do sistema.

Figura 5.5 Correspondência ou matching. Exibem-se as linhas que ligam os pontos-chave correspondentes entre duas imagens.

Figura 5.6 Corpo sujeito a uma deformação em $x$ : estado inicial e estado final. 
Figura 5.7 Imagem inicial (original, antes da deformação) e imagem final (após a deformação).

Figura 5.8 Deslocamento radial $[\mathrm{mm}]$ e deslocamento tangencial $[\mathrm{rad}]$.

Figura 5.9 Imagem inicial (antes da deformação) e imagem final (após deformação) em coordenadas polares.

Figura 6.1 Imagens de uma peça metálica com furo.

Figura 6.2 Localização de pontos-chave pelo SIFT.

Figura 6.3 Imagem original e final, depois da aplicação de deslocamentos bidirecionais.

Figura 6.4 Imagem original e final, depois da aplicação de deslocamentos bidirecionais.

Figura 6.5 Imagem criada artificialmente de uma placa com furo

Figura 6.6 Imagem final deformada após a aplicação de deslocamentos bidirecionais associados a uma tensão nominal $\sigma_{n}$ na direção horizontal.

Figura 6.7 Simulação da distribuição do campo de deformações de uma placa com furo central, para uma tensão nominal $\sigma=9 K N$.

Figura 6.8 Gráfico dos erros na medição visual da deformação associados à resolução.

Figura 7.1 Extensômetro Instron montado no corpo de prova.

Figura 7.2 Montagem experimental do corpo de prova cilíndrico de alumínio.

Figura 7.3 Montagem experimental do corpo de prova cilíndrico de aço, lixado para gerar texturização.

Figura 7.4 Curva tensão-deformação para o corpo de prova de alumínio, com deformações medidas pelo strain gage e pelo sistema visual. 
Figura 7.5 Curva tensão-deformação para o corpo de prova de aço, com deformações medidas pelo strain gage e pelo sistema visual.

Figura 7.6 Extensômetro colável da Excel Sensores Ltda de resistência elétrica, modelo roseta dupla a $90^{\circ}$.

Figura 7.7 Montagem experimental do corpo de prova.

Figura 7.8 Montagem experimental do corpo de prova.

Figura 7.9 Valores do coeficiente de Poisson, com deformações medidas pela roseta extensométrica (strain gage) e pelo sistema visual.

Figura 7.10 Valores do coeficiente de Poisson, com deformações medidas pela roseta extensométrica (strain gage) e pelo sistema visual. 


\section{Lista de tabelas}

Tabela 6.1 Resultados dos parâmetros de deformação para o caso de uma deformação uniforme.

Tabela 6.2 Resultados dos parâmetros de deformação para o caso de uma deformação variável.

Tabela 6.3 Geometria da placa simulada

Tabela 6.4 Propriedades mecânicas adotadas.

Tabela 6.5 Resultados da estimação dos parâmetros de deformação para diferentes resoluções virtuais.

Tabela 7.1 Geometria do corpo de prova circular

Tabela 7.2 Módulo de Elasticidade em diferentes níveis de deformação.

Tabela 7.3 Módulo de Elasticidade em diferentes níveis de deformação.

Tabela 7.4 Resultados experimentais do Modulo de Elasticidade para o aço e alumínio.

Tabela 7.5 Geometria do corpo de prova plano.

Tabela 7.6 Dimensões do extensômetro elétrico utilizado.

Tabela 7.7 Coeficiente de Poisson para diferentes níveis de deformação

Tabela 7.8 Relação de Poisson para diferentes níveis de deformação.

Tabela 7.9 Tempos de execução do programa desenvolvido. 